\title{
Semantic Grid Applications to Complex Satellite Mission Systems
}

\author{
M. Sánchez-Gestido ${ }^{\# 1}$, L. Blanco-Abruña ${ }^{\# 2}$, M. S. Pérez ${ }^{* 3}$, R. González-Cabero ${ }^{* 4}$, A. Gómez-Pérez ${ }^{* 5}$, Ó. Corcho ${ }^{\$ 6}$ \\ ${ }^{\#}$ Deimos Space S.L., Madrid, Spain \\ 'manuel.sanchez@deimos-space.com, 리 is.blanco@deimos-space.com \\ *Universidad Politécnica de Madrid, Madrid, Spain \\ 3mperez@fi.upm.es, ${ }^{4}$ rgonza@fi.upm.es, ${ }^{5}$ asun@fi.upm.es \\ ${ }^{\$}$ University of Manchester, Manchester, $U K$ \\ 'oscar.Corcho@manchester.ac.uk
}

\begin{abstract}
This paper presents the application of a semantic grid architecture into a scenario for the product analysis of a representative Earth Observation Satellite Mission (EnviSat). This Use Case aims at demonstrating the benefits of a Semantic Grid approach to real world problems in terms of flexibility, reduction of SW running costs, maintainability, expandability, interoperability and definition of a standardized approach.
\end{abstract}

\section{INTRODUCTION}

Earth Observation can be defined as the science of getting data from our planet by placing in orbit a Hardware/Software element with several observation instruments, whose main goal is to obtain measurements from the Earth surface or the atmosphere. These scientific data are sent to Ground Stations and then processed in order to get meaningful information (i.e. images).

In a nutshell and putting aside other aspects, basic working of an Earth Observation Satellite System consists in a simple process that is repeated over time. The instruments on board the satellite act like cameras that can be programmed (very complex cameras nevertheless), taking "pictures" (images) of specific parts of the Earth at predefined times. Parameters for taking this pictures (like any camera would need to operate) and also parameters for the satellite general configuration, constitute the information included in the Mission Planning issued by the Mission Planning System (MPS), which is sent to the Flight Operation Segment (FOS), that in turn sends an equivalent information to a Ground Station and from there to the satellite.

FOS converts the information in the Planning into the shape of MacroCommands (MCMD's). This means a translation from one planning format to another that is meaningful to the satellite. The Ground Station translates the MCMD information into a RadioFrequency link to communicate with the satellite antenna in the Service Module of the spacecraft. When MCMD's are transferred in a radiofrequency link they are generically called TeleCommands, TC.

Computer on board the satellite will store the list of MCMD's, each of them with a time tag that marks the execution time of that MCMD. Taking one "picture" would mean, for instance, the execution of a MCMD that copies those parameters for taking the picture, from the memory of the satellite computer to the instrument computer memory, and then the execution of the MCMD that triggers the camera shot.

"Pictures" from each of the instruments are stored onboard (in the satellite computer memory) as raw data and when the satellite over-flies the Ground station that information is sent to the Ground Station antenna (Data downlink). In that Ground Station a preliminary conversion from raw data to a so-called "Level 0 " product is performed (basically adding a identification label to each of the pictures). These "Level 0" products are sent to the Payload Data Segment (PDS) that generate Level $1 b$ (with geo-location data) and the final Level 2 products, which are made available to the final user community (scientist, environmental organizations, etc). A more detailed explanation of the whole system can be found in [1].

\section{A SEMANTIC GRID INFRASTRUCTURE FOR THIS SCENARIO}

The described use case involves a complex process in which distributed data belonging to different facilities and organizations must be queried, processed and transferred. Suitable access control mechanisms have to be defined for these operations. Currently, the location of the resources and the processing of data are made in a wired way, according to filenames and content of these files in an "ad-hoc" format.

The use of a grid framework provides a flexible way of locating required resources and the virtualisation of these resources by means of Grid Services. Besides, there is a need for managing lifetime of certain data resources, since there are data changes along the lifetime of the system.

Several resources used in this use case benefit from the generation and use of semantically-rich metadata, which is made up by means of an automated annotation process. This process is needed to link file resources with their actual content meaning (that is, to provide metadata about the data used in the system). Not only content should be annotated, but also provenance information, including temporal information (time stamp) about the data and the computational tasks involved in their generation. The explicit and formal relationships between operational parameters of the satellite instruments and the data obtained from them can also be represented in an ontology, and annotated from the actual 
planning of the instruments.

Fig. 1 presents a general overview of the satellite process, with a centralized ontology describing the concepts and relationships between elements. Data (planning and product files) and associated metadata are completely distributed in a number of sub-facilities, breaking up the demands in terms of computational resources and disk storage. Within this distributed layout it is possible to perform queries on the annotated metadata, allowing the system to retrieve information and build up more elaborated reports and plots which are eventually presented for the user.

During nominal operation of a Satellite Mission, external facilities are generating files on a continuous basis, with a frequency that varies from file type to file type. Due to the enormous amount of files circulating in the system and the huge size on the final products, the system could perform the annotation process on these files at different levels. On a regular basis, and for every arriving file, some basic information is annotated (e.g. time span for the data contents, instrument data, processing facility). Later on, and only in association with a query performed by the user, a more complete annotation can be performed for the contents of the particular files relevant to the output of the query. This ondemand annotation process reduces the amount of metadata stored in the system to the actual needs of the user.

Regarding security, the involvement of several organizations implies the establishment of different access policies and the definition of Virtual Organizations (VO). The role of each specific actor within an organization also defines its privileges as a member of the VO. A VO prototype developed as part of the OntoGrid project has already proved that VO's can be created and dismantled. In the context of Satellite Missions, this is of use in the event, for instance, of an instrument failure in orbit. In some occasions, this incident requires setting up a specific team gathering expertise from many different areas. When an instrument has a severe failure (hopefully not many times during its lifetime), it goes to Safe mode and it is probably necessary to put together: a mission analysis expert, the instrument responsible, a senior satellite operations engineer and maybe the payload manager. In order to analyse what happened and to define a strategy to recover the instrument, it is helpful to gather as much organized information (in relation to the incident) as possible. It should be noted that when the instrument is in orbit no direct access (for observation or repair) is possible. All the analysis has to be done on the basis of the information that is retrieved from different parts of the system of what happened around the time of the failure. Easy access to this information (structured, and defining visualization) is useful to gradually recover the instrument from Safe Mode.

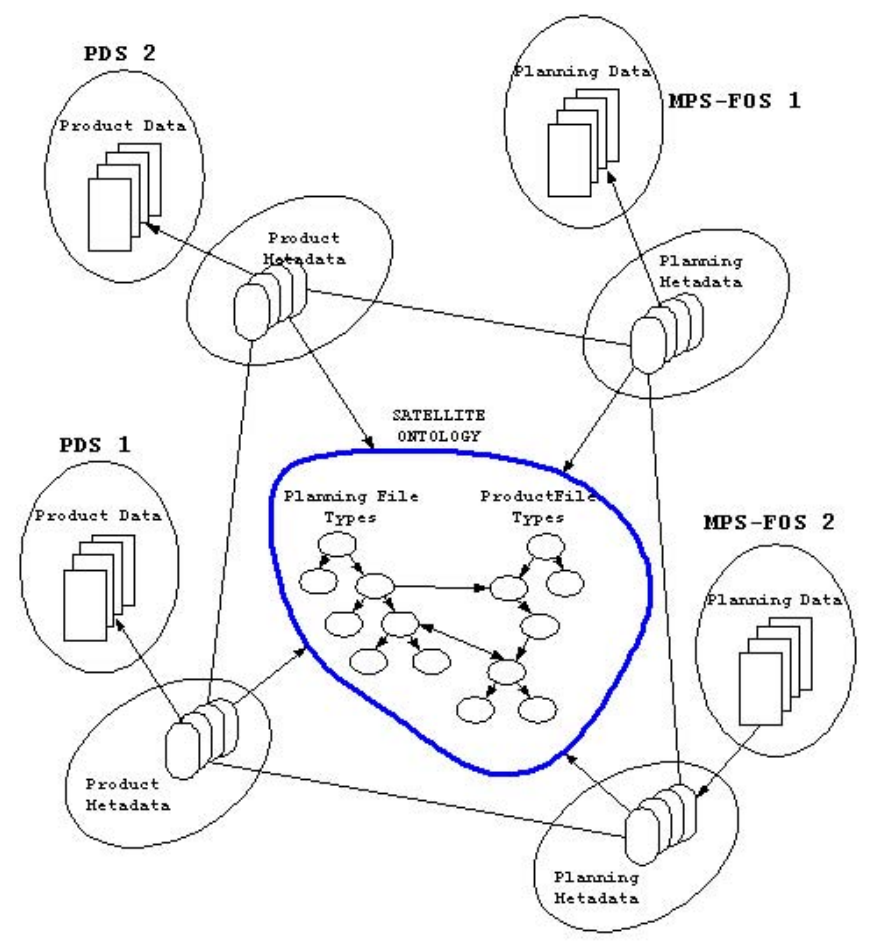

Fig. 1. Semantic Grid infrastructure for distributed file annotation and metadata queries

\section{CONCLUSIONS}

This paper has addressed the benefits of the application of a semantic grid architecture in a complex environment, such as a satellite mission system. This architecture, named S-OGSA, is being developed in the Ontogrid project. The lessons learn from this task can help us to extend this work to other different satellite missions.

\section{ACKNOWLEDGMENT}

The authors would like to thank the other members of the OntoGrid consortium for many thoughtful discussions. The authors are also very grateful to the European Space Agency (ESA) representatives Olivier Colin (ESA-ESRIN) and Pierre Viau (ESA-ESTEC) for providing access to the actual products and auxiliary tools from the Envisat mission.

\section{REFERENCES}

[1] M. Sánchez Gestido. "OntoGrid Business Case and User Requirements Analysis and Test Set Definition For Quality Analysis of Satellite Missions". October 2005. OntoGrid Deliverable D8.1. Available: http://www.ontogrid.net 